\title{
TEATRO CLÁSICO Y TRADICIÓN TEXTUAL: UNA PROPUESTA DE EDICIÓN CRÍTICA DIGITAL
}

\section{CLASSICAL THEATRE AND TEXTUAL TRADITION: A PROPOSAL FOR A DIGITAL CRITICAL EDITION}

\author{
Marco Presotto \\ Università di Bologna \\ marco.presotto@unibo.it
}

Sònia Boadas

Universitat Autònoma de Barcelona

sonia.boadas@gmail.com

\begin{abstract}
Resumen
El artículo ofrece unas breves consideraciones sobre el panorama actual de los corpus textuales digitales del teatro clásico europeo, con un especial enfoque hacia el contexto español. Dentro de este ámbito, se pone énfasis en el proceso de realización y las características de la edición crítica y el archivo digital de una comedia española del siglo XVII, La dama boba de Lope de Vega. Este proyecto, que se ha realizado con fines experimentales, pretende ofrecer a la comunidad científica un posible modelo de representación de tradiciones textuales complejas que implique la superación de los límites de la edición tradicional en papel.
\end{abstract}

Palabras clave: Edición crítica. Teatro. Lope de Vega. Archivo digital. La dama boba. 


\begin{abstract}
The article provides a brief consideration about the current presence of digital textual corpora of classical European theatre, with a special focus on Spanish context. Within this area, it describes the production process and the features of the critical edition and digital archive of La dama boba, a Spanish play of the seventeenth century written by Lope de Vega. This project - that has been carried out with experimental purposes- aims to offer to the scientific community a possible model to represent complex textual traditions involves overcoming the limits of the traditional paper editions.
\end{abstract}

Keywords: Critical Edition. Theatre. Lope de Vega. Digital Archive. La dama boba.

\title{
1. EDICIONES DIGITALES Y TEATRO CLÁSICO EUROPEO
}

La imponente producción teatral que se desarrolló en Europa en los siglos XVI y XVII cuenta con coincidencias sorprendentes entre los distintos ámbitos nacionales ${ }^{1}$. A pesar de las notables diferencias de contexto social, expectativa estética y exigencias del mercado, es patente el aprovechamiento común de modelos formales, fuentes literarias y estrategias de organización del texto-espectáculo. Esta visión europea del fenómeno teatral clásico se está consolidando de manera sistemática en las comunidades científicas ${ }^{2}$, aunque debe resolverse en primer lugar la dificultad objetiva de facilitar el acceso a los corpus textuales para la comparación. Bajo este aspecto, es una iniciativa muy innovadora la propuesta de Canon 60 por parte del macroproyecto TC/12 dirigido por Joan Oleza, que ha publicado la edición digital en formato HTML de una selección de 60 obras clásicas del teatro español, con la aspiración de hacer visible internacionalmente el núcleo más valioso del patrimonio teatral clásico español a través de las herramientas digitales. El mismo investigador, además, ha realizado otra biblioteca digital llamada EMOTHE (The Classics of Early Modern European Theatre), que propone un repertorio de textos teatrales antiguos españoles, ingleses, italianos y franceses en edición fiable y con traducciones en las principales lenguas implicadas ${ }^{3}$. Por otro lado, se está afianzando la tendencia a realizar ediciones completas en línea del teatro de los

\footnotetext{
${ }^{1}$ El presente trabajo se inscribe en el Proyecto de Investigación FFI2012-35950, Edición y estudio de 36 comedias de Lope de Vega, financiado por el Ministerio de Economía y Competitividad.

${ }^{2}$ Nos limitamos a señalar aquí, a manera de ejemplo el congreso sobre Lope de Vega y el teatro europeo de su tiempo, organizado por el grupo Prolope de la Universitat Autònoma de Barcelona y celebrado en diciembre 2015, cuyas actas se publicaron en el Anuario Lope de Vega (volumen 23, 2017), accesible desde http://revistes.uab.cat/anuariolopedevega/index. También el grupo de investigación Proteo de la Universidad de Burgos organizó el congreso Teatro español y Teatro europeo: relaciones bilaterales, en Burgos, del 14 al 16 de diciembre de 2016.

${ }^{3}$ Accesible desde http://emothe.uv.es/biblioteca/index.php?.
} 
mayores dramaturgos europeos desde una perspectiva nacional, con esfuerzos a veces titánicos y resultados excelentes. Es comprensible la oscilación de los productos digitales entre un objetivo prevalentemente divulgativo (con una mayor o menor atención hacia la historia del teatro o de la literatura dramática) y la finalidad puramente científica, relacionada sobre todo con los problemas de la tradición textual, las variantes y los testimonios en su materialidad (manuscritos autógrafos, impresos antiguos, etc.).

Pueden citarse aquí algunos ejemplos significativos a partir de la labor en torno a William Shakespeare, cuya fama global atrae mayores recursos. La Internet Shakespeare Edition de la University of Victoria (Canadá) ${ }^{4}$ es un proyecto monumental que consigue equilibrar las dos perspectivas indicadas, dado que se propone como un one-stop shop en constante actualización, que se ocupa tanto de los aspectos filológicos como del contexto histórico y de la puesta en escena. Su vertiente didáctica, enfocada principalmente a la enseñanza, la convierte en una herramienta de uso masivo entre la comunidad de internautas ${ }^{5}$ : los itinerarios de lectura que posibilita este instrumento modélico son amplísimos y cubren, a través de enlaces muy ilustrativos, todas las áreas del conocimiento implicadas en la comprensión del fenómeno artístico y cultural. En cuanto al aspecto específico de la edición de las obras, el proyecto ofrece un sistema muy extenso de contenidos a partir de una introducción detallada de cada pieza, un apartado de contextual materials de gran utilidad, el texto modernizado con una amplia anotación para facilitar su comprensión, una transcripción no modernizada y también los facsímiles de los testimonios utilizados, que pueden consultarse de forma sinóptica.

Un proyecto en parte comparable en el mundo francófono, aunque de menor extensión, es Toutmoliere, realizado por el Ayuntamiento de Pézenas. El portal ofrece ediciones fiables de las obras de Molière con una amplia anotación y una variedad de apartados complementarios con fines divulgativos ${ }^{6}$. Más expresamente volcado a un uso científico es, en cambio, Moliere 21, dirigido por George Forestier de la Université de la Sorbonne ${ }^{7}$ : en este caso prima el interés hacia la investigación del corpus, a través de una interesante base de datos intertextual y la comparación entre las transcripciones de los testimonios de la tradición textual, a menudo marcados por los procesos de censura, con el objetivo de ofrecer una herramienta complementaria a la edición tradicional en papel ${ }^{8}$.

\footnotetext{
${ }_{5}^{4}$ Accesible desde http://internetshakespeare.uvic.ca/.

${ }^{5}$ Como bien se expresa en su sitio web: "In a busy month, our server receives requests from around the world for over 3.5 million pages, and many visitors stay to examine dozens of pages. While our audience is global, our statistics show a high proportion of visitors from educational institutions" (http://internetshakespeare.uvic.ca).

${ }^{6}$ Así se declara en su web: "Ce site, conçu dans un esprit de rigueur scientifique, est destiné à un public large. Aux étudiants ou lycéens qui recherchent des éléments d'information relatifs à Molière; aux curieux et aux amateurs de théâtre qui s'intéressent à notre dramaturge, et aux chercheurs qui y trouveront un certain nombre d'outils de travail commodes" (http://www.toutmoliere.net/).

${ }_{7}^{7}$ Accesible desde http://www.moliere.paris-sorbonne.fr/.

8 "Le projet MOLIERE21 se fixe pour objectif de procurer une édition 'mixte' des OEuvres de Molière, tirant parti des avantages respectifs du support électronique en ligne et du support imprimé, conformément aux principes régissant
} 
Entre las aplicaciones para dispositivos móviles, las elaboraciones más complejas se han realizado en el ámbito del teatro de Shakespeare y son de corte didáctico y divulgativo. Reviste especial interés The Folger Luminary Shakespeare ${ }^{9}$, caracterizado, entre otros aspectos, por un enfoque hacia la declamación del texto, cuyo audio resulta disponible a veces en diferentes grabaciones históricas. El proyecto, en su conjunto, constituye una herramienta dirigida a implementar la coparticipación de los usuarios y la creación de comunidades de estudio y lectura.

En este panorama, el contexto español se ha desarrollado de una manera peculiar. A causa posiblemente del inabarcable patrimonio textual que constituyen los textos dramáticos del Siglo de Oro, solamente en parte conocidos y editados con criterios ecdóticos, los grandes proyectos digitales han privilegiado la realización de repositorios, con afán cuantitativo ${ }^{10}$, y en menor grado de la construcción de complejos instrumentos de búsqueda y selección ${ }^{11}$. La magna y meritoria empresa de la Biblioteca Virtual Miguel de Cervantes ${ }^{12}$ recoge una impresionante cantidad de obras teatrales, disponibles a veces a través del facsímil de la edición antigua, en otros casos, transcritas a partir de ediciones modernas autorizadas o de un testimonio del siglo XVII. Actualmente, se trata del principal archivo disponible en forma gratuita, aunque los criterios de edición no son uniformes y la fijación del texto a través del estudio de las variantes no está prevista en este contexto.

En el caso de Lope de Vega, representa una novedad absoluta en el panorama digital el proyecto Artelope ${ }^{13}$ dirigido por Joan Oleza, que pone a disposición del investigador una base de datos de los argumentos del Teatro del Fénix de los Ingenios (constituido por más de cuatrocientas piezas) con apartados complementarios de gran utilidad; entre ellos, ofrece ediciones a cargo de miembros de su equipo. Tampoco aquí es prioritaria la atención a la fijación del texto con criterios ecdóticos (se utilizan principalmente ediciones o transcripciones

les autres projets du CEPM", accesible desde http://moliere.paris-sorbonne.fr/objectifs.php. La sigla CEPM se refiere al "Corpus Électroniques de la Première Modernité" (http://www.cepm.paris-sorbonne.fr/).

9 Accesible desde http://www.folger.edu/folger-luminary-shakespeare-apps. El equipo remite a la Folger Shakespeare Library en Washington (http://www.folger.edu/), que también pone a disposición de forma gratuita los textos digitales en varios formatos (PDF, XML, HTML, DOC, TXT).

${ }^{10}$ Hasta la fecha, la única base de datos realmente manejable como conjunto para búsquedas avanzadas es TESO. Teatro Español del Siglo de Oro (http://teso.chadwyck.co.uk/). Es un producto comercial publicado en 1997 muy difundido en la comunidad científica. Contiene 800 piezas dramáticas transcritas directamente de testimonios antiguos y sin criterios ecdóticos. Cabe recordar también la grata y pionera labor de la Association for Hispanical Classical Theatre (http://www.wordpress.comedias.org/) que ofrece una notable cantidad de textos digitales editados principalmente gracias al esfuerzo de Vern Williamsen, ahora bajo la coordinación de Matthew Stroud (http://www.comedias.org/textlist.html).

${ }^{11}$ Tiene una perspectiva metodológica más específica el interesante portal Teatro de los Siglos de Oro dirigido desde 1995 por Ricardo Serrano y Alfredo Hermenegildo, albergado por la Université du Québec à Trois Rivières (http://uqtr.ca/teatro/entrada/t.html). El recurso pone a disposición del lector 34 piezas teatrales en varios formatos, así como una herramienta de búsqueda avanzada, El Brocense, que permite "formatar [sic], buscar, contar, segmentar según diferentes criterios, visualizar las presencias de los personajes en escena (desde un punto de vista actoral, por ejemplo) y aislar los actos de conversación entre personajes". Entre las ediciones publicadas, cabe señalar la propuesta de edición paralela de La verdad sospechosa de Alarcón y Le menteur de Corneille a cargo de Ricardo Serrano.

${ }^{12}$ Accesible desde http://www.cervantesvirtual.com/.

${ }^{13}$ Accesible desde http://artelope.uv.es/. 
anteriores, aunque revisadas de manera rigurosa), pero lo que se ha realizado hasta ahora (110 textos editados) empieza a representar un importante corpus coherente desde el punto de vista formal y aprovechable en el futuro para su estudio de conjunto. Por su enfoque hacia el texto, reviste mayor interés el proyecto de edición electrónica, aunque con un explícito interés didáctico y divulgativo, de La traición en la amistad de María de Zayas, dirigida por Teresa Ferrer (2015) de la Universitat de València ${ }^{14}$. La edición se ha realizado con una perspectiva tradicional que recuerda la del libro en papel, pero propone un comentario de texto que se reparte en varias tipologías de anotación, fácilmente reconocibles, que responden idealmente a diferentes necesidades de lectura. Se trata de un texto muy enriquecido, que cuenta con la inserción de imágenes, video y sonidos. Además, el libro está disponible también en formato para dispositivos móviles.

Estos pocos ejemplos escogidos sin afán de exhaustividad dan cuenta de las diferentes perspectivas metodológicas que presuponen distintas competencias y exigencias del usuario: frente a una tradición editorial en papel asentada sobre criterios preconcebidos de definición del mercado, construidos a partir de una supuesta recepción estudiantil universitaria y erudita, el soporte informático parece imponer nuevamente al editor digital una mayor atención hacia su lector ideal y al contexto de utilización de la herramienta en su conjunto.

\section{LA EDICIÓN CRÍTICA DIGITAL DE LA DAMA BOBA}

En el contexto del equipo de investigación Prolope, que desde 1989 se dedica al estudio y publicación de las comedias de Lope de Vega, nació hace algunos años la idea de realizar un proyecto de edición crítica digital que, gracias al soporte informático, pudiera facilitar la visión, consulta y lectura de varios testimonios de la transmisión textual de una comedia, para realizar un archivo digital complementario, a la labor editorial propiamente dicha. La intención era crear un posible modelo aprovechable, para todas las tradiciones textuales complejas del teatro de Lope de Vega, y dar así, el justo relieve a informaciones de interés para la comunidad científica. Como resultado de esa idea inicial, se presenta aquí el proyecto La dama boba: edición crítica y archivo digital (Presotto, 2015), fruto de la colaboración entre el grupo de investigación Prolope de la Universitat Autònoma de Barcelona y el CRR-MM (Centro di Risorse per la Ricerca Multimediale) de la Università di Bologna. El proyecto se propone ofrecer una página web dedicada a la comedia La dama boba, que permita la lectura de la edición crítica de la obra y que ofrezca, por primera vez, un archivo de

\footnotetext{
${ }^{14}$ Accesible desde http://dicat.uv.es/te@doc/edicion/TraicionenAmistad_Zayas.html.
} 
los testimonios utilizados y su transcripción, así como varias opciones de visualización y de búsqueda.

La comedia La dama boba conoce ya ediciones digitales que se pueden consultar gratuitamente en línea, con herramientas de búsqueda o de visualización automática de aspectos estructurales. La Biblioteca Virtual Miguel de Cervantes pone a disposición del usuario una versión en formato HTML de la edición de Alonso Zamora Vicente $(1946)^{15}$, con la opción de búsqueda de concordancias, mientras que, el proyecto Artelope ofrece una versión mejorada de esta misma edición, marcada digitalmente por Eva Soler Sasera (20132014), en la cual se han implementado algunas funciones como evidenciar las acotaciones, los apartes o la métrica ${ }^{16}$. Sin embargo, estas ediciones no conllevan una aproximación a la compleja historia textual de la pieza, que es el objetivo prioritario del grupo de investigación Prolope y de este proyecto en particular.

Han sido varios los proyectos que durante los últimos años han prestado especial atención a los manuscritos autógrafos y a la transmisión textual del texto, y que nos han ayudado a concebir La dama boba: edición crítica y archivo digital. Mención especial merecen el Jane Austen's Fiction Manuscripts (Sutherland, 2010), desarrollado entre la University of Oxford y el King's College de London, que pretende crear un repositorio digital donde reunir todos los manuscritos autógrafos de ficción de Jean Austen, proporcionando también su transcripción y análisis ${ }^{17}$; el proyecto de edición digital y anotada de La entretenida de Cervantes, llevado a cabo por John O'Neill (2007), dentro del proyecto Out of the Wings, con la colaboración del Departamento de Español y del Departamento de Humanidades Digitales del King's College ${ }^{18}$. Después de las primeras incursiones del grupo de investigación Prolope en el mundo de las Humanidades Digitales con la publicación del libro virtual de Mujeres y criados (Boadas y Valdés, 2014), a raíz del descubrimiento de esta comedia inédita de Lope de $V_{\text {ega }}{ }^{19}$, el proyecto que presentamos pretende ofrecer una lectura fiable y autorizada de La dama boba en formato digital, que a su vez permita una lectura más personalizada, con herramientas de navegación y un sistema de búsqueda avanzado.

\footnotetext{
${ }^{15}$ Accesible desde http://www.cervantesvirtual.com/obra/la-dama-boba--0/.

${ }_{17}^{16}$ Accesible desde http://artelope.uv.es/biblioteca/textosAL/AL0575 LaDamaBoba.php.

${ }_{17}$ Los objetivos fundamentales del proyecto son: "To provide for the first time full descriptions of, transcriptions of, analysis of, and commentary on the manuscripts in the archive, including details of erasures, handwriting, paper quality, watermarks, ink, binding structures, and any ancillary materials held with the holographs as aspects of their physical integrity or provenance" y "to develop complex interlinking of the virtual collection to allow systematic comparison of the manuscripts under a number of headings representing both their intellectual and physical states" (http://www.janeausten.ac.uk/about/overview.html).

18 "This edition offers a new perspective on Cervantes as a playwright by providing four views of the Spanish text, together with an English translation, and by presenting the text in a way that privileges the verse, thus acknowledging the importance of poetic form in Spanish Golden Age drama. The edition also incorporates various indices that help to contextualise the play, as well as features designed to aid theatre practitioners, such as menus that enable one to quickly access costume, props and stage furniture requirements for each scene, and to track the speeches and movements of individual characters" (http://entretenida.outofthewings.org/aboutproject/index.html).

${ }^{10}$ El libro virtual Mujeres y criados, accesible desde http://online.fliphtml5.com/tbai/hyew/ - p=8.
} 
En una visión panorámica del proceso de definición del texto crítico, hemos considerado necesario experimentar con instrumentos que permitan establecer una relación más estrecha entre los documentos y su transcripción, de cara a una visualización ideal de las características materiales de la tradición textual. Todo ello tiene el objetivo de ofrecer al lector los resultados de la investigación y también la información sobre el trabajo científico realizado. De esta manera se puede dar un salto cualitativo importante en las ediciones críticas: pasamos de tener el texto fijado con su aparato de variantes, a la posibilidad de visualizar directamente los testimonios, con su transcripción, confrontables entre ellos y con la edición crítica.

Esta ambición de carácter ecdótico del proyecto está relacionada con las características materiales de la tradición textual del teatro del Siglo de Oro, cuyos textos se caracterizan por su inestabilidad y en la que el concepto mismo de texto debe replantearse constantemente en relación con el medio. No es infrecuente encontrar piezas cuya tradición textual revela la existencia de lo que podríamos denominar diferentes versiones de una misma obra a raíz de la cantidad y diversidad de variantes recogidas tras la collatio. En estos casos, el editor crítico está obligado a elegir el texto base y relegar las variantes al aparato a pie de página o a apartados específicos, facilitando así la lectura de la obra según existió en un determinado momento y bajo ciertas circunstancias, o bien tomar la decisión salomónica de publicar diferentes versiones, lo cual no resuelve el problema ecdótico, pero pone a disposición del lector interesado todos los documentos. Este es el caso de piezas importantes de este legado artístico, como la misma Numancia de Cervantes o algunas de las comedias de Lope de Vega, como La bella malmaridada, publicada en la Segunda parte de comedias. La reflexión sobre este tema ha sido constante a lo largo de los años, y las herramientas informáticas deben constituir una posible salida de este impasse editorial.

La elección de La dama boba para llevar a cabo este proyecto experimental no ha sido casual: se trata de una de las comedias más conocidas y representadas del teatro cómico de Lope de Vega, y que presenta además una compleja tradición textual. Conservamos el manuscrito autógrafo $(\mathrm{O})$ de la obra fechado en 1613 con intervenciones del mismo autor que permiten apreciar la historia de la génesis de la pieza, los pentimenti, las construcciones in itinere o las correcciones a posteriori. La edición crítica tradicional, con sus exigencias y limitaciones también derivadas del soporte en papel, reduce considerablemente la atención a todos estos aspectos, relegándolos generalmente, a un apéndice o a una síntesis introductoria, unos aspectos que a través de una edición digital pueden recobrar toda su relevancia. También ha llegado hasta nosotros, la edición autorizada (A) que apareció en 1617 dentro de la Novena parte de comedias (Madrid, Viuda de Alonso Martín), publicada por el dramaturgo cinco años después de haber redactado la obra, sin tener acceso al autógrafo. Además, conservamos un manuscrito con letra del siglo XVII que deriva de un proceso de 
memorización de los conocidos como memoriones (M), personas que asistían varias veces a la representación de una comedia y que hurtaban el texto de la misma para venderla a compañías menores o a lectores interesados. Esta compleja tradición textual hace de La dama boba una comedia idónea para explotar las posibilidades que las Humanidades Digitales ofrecen a los estudios de crítica textual.

\subsection{Codificación XML-TEI}

Por lo que se refiere a los aspectos técnicos del proyecto, para el marcaje de la comedia, se eligió el sistema de codificación XML-TEI, uno de los estándares de marcado más utilizados que permite la portabilidad de documentos independientemente del software utilizado, y con unas potencialidades de explotación del texto muy elevadas. Se definieron las características imprescindibles que debían marcarse en el caso del manuscrito autógrafo, cuya transcripción debía dar cuenta de las intervenciones, los pentimenti y las correcciones in itinere de Lope de Vega, en un intento de reconstruir el orden y la intención con que se produjeron. En este caso concreto, no estamos solo ante el marcado de unas intervenciones, marcado que además resulta en algunos casos ciertamente técnico y complejo, sino que detrás de las etiquetas de codificación hay una interpretación filológica de los pasajes, ya que es el editor quien ofrece su visión sobre el proceso de escritura de Lope de Vega.

Está claro, en todo caso, que esta fase de transcripción y de codificación es mucho más larga y compleja respecto a lo usual, y que requiere por parte del filólogo de un conocimiento específico del lenguaje de marcado XML-TEI, y en particular de las etiquetas del módulo sobre la representación de las fuentes primarias.

\subsection{AnnotElted}

Para realizar la codificación del texto, la coordinación técnica del proyecto diseñó AnnoTElted, una plataforma de producción de archivos TEI/XML que prevé el uso de un rich markup editor realizado en el entorno wiki para la transcripción y el marcaje del texto, con la visualización contemporánea del facsímil de los testimonios. 


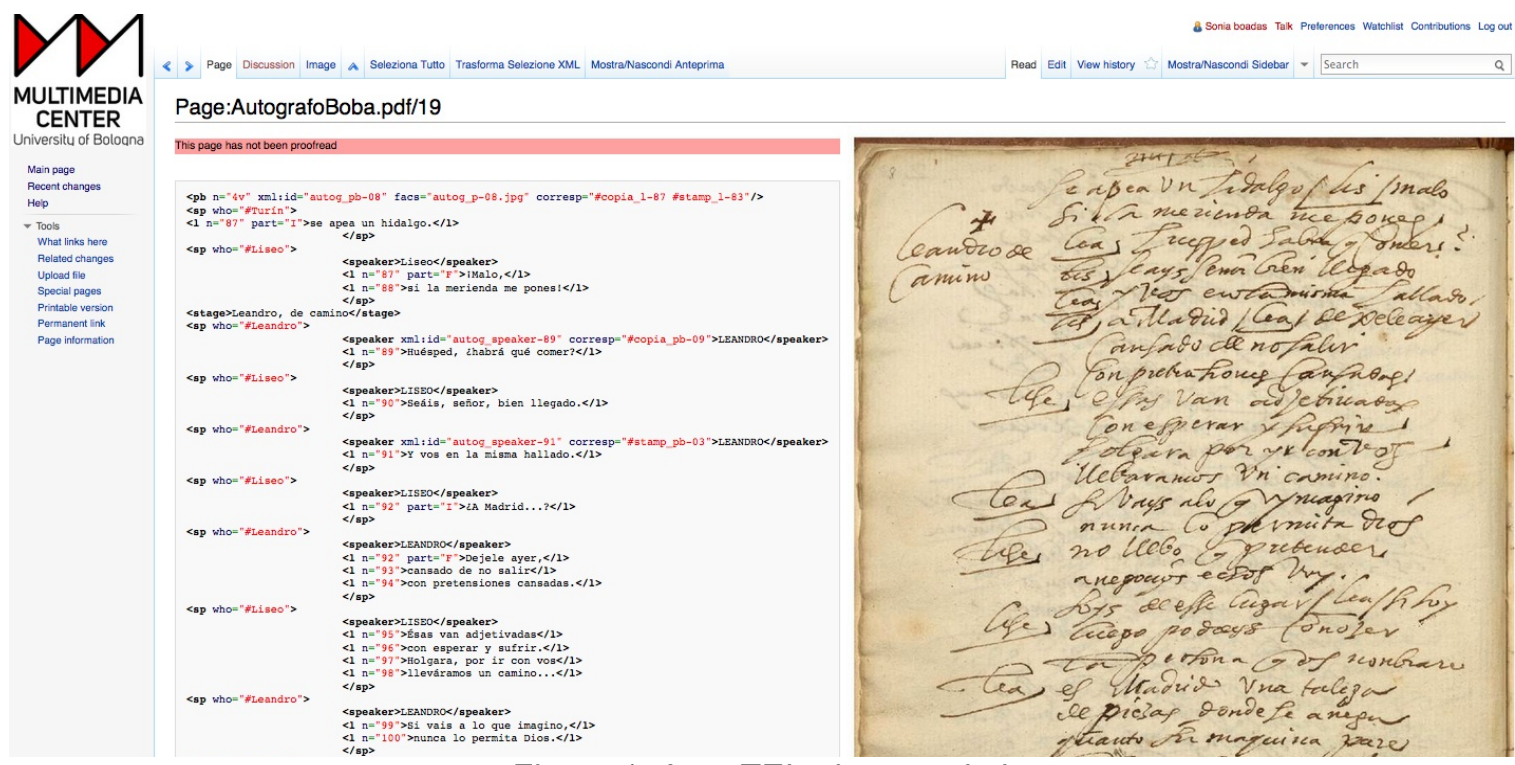

Figura 1. AnnoTElted en modo lectura.

El carácter colaborativo de esta plataforma permite la transcripción y codificación de los archivos en equipo y de forma remota. Mediante unas credenciales personales, los colaboradores del proyecto han podido acceder simultáneamente al editor para codificar los documentos, pudiéndose repartir el trabajo, ya fuera por segmentos de la obra o por tipologías de intervención. Asimismo, la plataforma colaborativa diseñada ad hoc tiene la ventaja de acelerar el marcado gracias a la creación de iconos específicos para cada necesidad concreta del documento (<sp>, <speaker $>$, <stage>, <del>, <add>, entre otros), al estilo de los principales editores TEI comerciales.

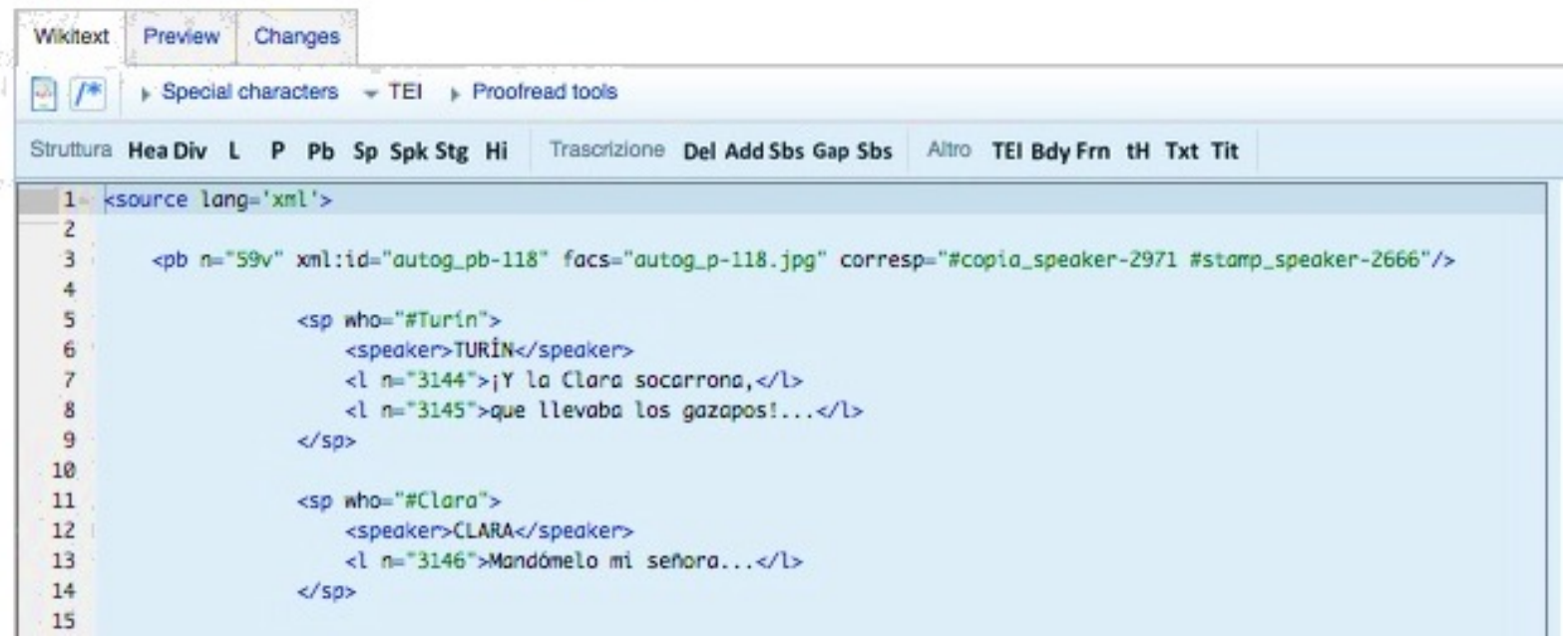

Figura 2. Detalle AnnoTElted modo edición.

La posición del facsímil en la visualización, así como su dimensión, se establecen según las necesidades de lectura. La plataforma incorpora también un sistema de validación, parcial o completa, de la página que permite averiguar el resultado de la transcripción y 
evidenciar posibles errores en una preview en HTML. Para las características de este proyecto, las principales ventajas de la plataforma AnnoTElted tienen que ver con la posibilidad de realizar un trabajo colaborativo, contemporáneo y de forma remota con backup automático, además de la posibilidad de personalizar las funciones a partir de las principales acciones previstas y la opción de tener la previsualización inmediata de la transformación de XML a HTML.

Asimismo, la plataforma permite reconstruir la cronología de la codificación que se ha realizado a través de la opción view history, que nos indica todas las intervenciones que ha habido en la página ordenadas por fecha. Seleccionando dos versiones distintas, se ofrece una comparación de las mismas para saber qué modificaciones se han introducido, tanto añadidos como supresiones, y quién las ha incorporado.

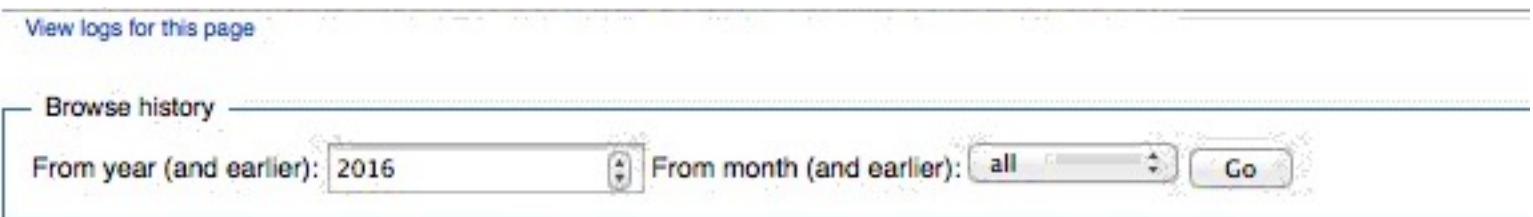

Diff selection: Mark the radio boxes of the revisions to compare and hit enter or the button at the bottom. Legend: (cur) $=$ difference with latest revision, $(\mathbf{p r e v})=$ difference with preceding revision, $\mathbf{m}=$ minor edit.

Compare selected revisions

- (cur I prev) ๑ 17:59, 28 September 2015 Marco Presotto (Talk I contribs) .. (3,488 bytes) (0) ... (undo)

- (cur I prev) 0 O 19:39, 24 September 2015 Marco Presotto (Talk I contribs) . . (3,488 bytes) (0) . . (undo)

- (cur I prev) $\bigcirc 0$ 16:01, 20 July 2015 Marco Presotto (Talk I contribs) .. (3,488 bytes) (+1) . . (undo)

- (cur I prev) $\bigcirc \bigcirc$ 19:59, 6 March 2015 Marco Presotto (Talk I contribs) . . (3,487 bytes) (+123) . . (undo)

- (cur I prev) 0 ○ 11:28, 9 February 2015 Eugenio Maggi (Talk I contribs) . . (3,364 bytes) (+39) . . (undo)

- (cur I prev) $\bigcirc \bigcirc$ 17:14, 5 February 2015 Aurelia Pessarrodona (Talk I contribs) .. (3,325 bytes) (+45) . . (undo)

- (cur I prev) 0 16:42, 2 February 2015 Sonia boadas (Talk I contribs) . . (3,280 bytes) (+53) . . (undo)

- (cur I prev) 0 0 09:53, 12 November 2014 Sonia boadas (Talk I contribs) . . (3,227 bytes) (+76) . . (undo)

- (cur I prev) $\bigcirc \bigcirc$ 09:51, 12 November 2014 Sonia boadas (Talk I contribs) . . (3,151 bytes) (-830) . . (undo)

- (cur l prev) $\odot \quad 09: 49,12$ November 2014 Sonia boadas (Tak l contribs) .. (3,981 bytes) $(+3,981)$. . ( $\rightarrow$ Da rivedere) Compare selected revisions

Figura 3. Cronología de las intervenciones. 
Revision as of 09:49, 12 November 2014 (edit) Sonia boadas (Talk I contribs) $(\rightarrow$ Da rivedere)
Latest revision as of 17:59, 28 September 2015 (edit) (undo) Marco Presotto (Talk | contribs)

(8 intermediate revisions by 4 users not shown)

Line 1:

enoinclude $>$ «pagequality level="2" user="Sonia boadas" |>div class="pagetext" >

esp who=" \#Otavio"s

$<$ speaker>OTAVIO</speaker

Line 17:

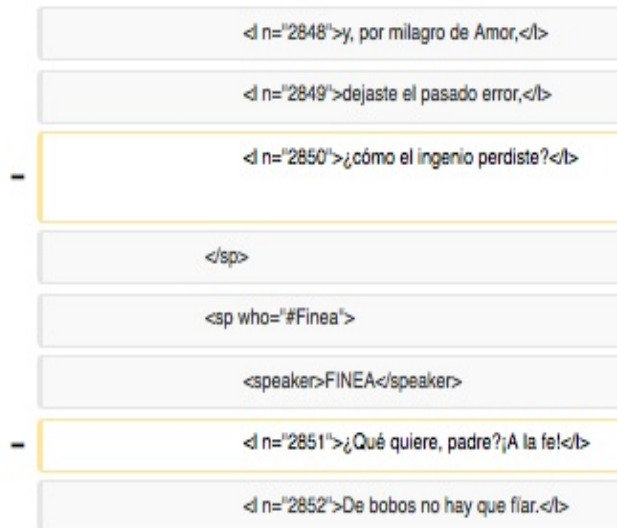

Line 1:

<noinclude $\times$ pagequality level="2" user="Sonia boadas $1 \rtimes<d v$ class="pagetext">

$+$

$+$

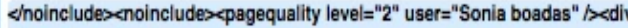

class="pagetext's

$<p b n=" 55 r$ " xml:id="autog_pb-109" facs="autog_p-109.jpg"

corresp $=$ "\#copia_speaker-2686 "stamp_speaker-2441"/s

$\langle$ sp who $="$ "Otavio"

speaker OTAVIO_ispeaker

Line 21:

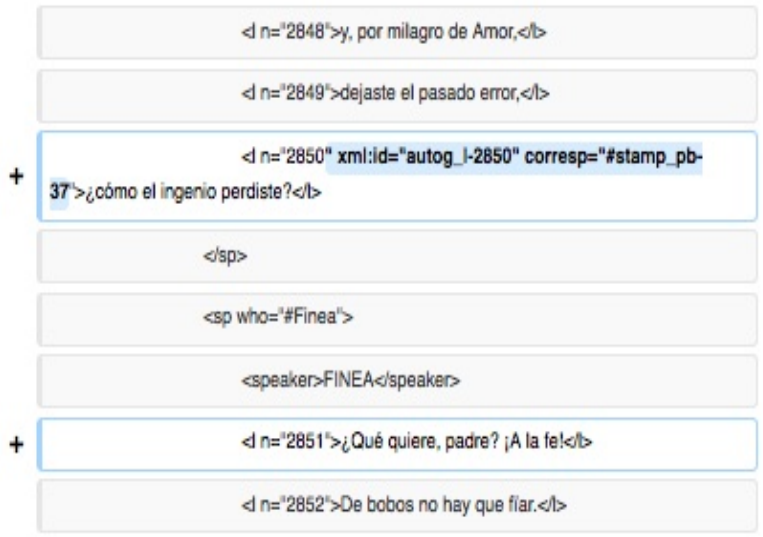

Figura 4. Comparación de las intervenciones.

Una vez realizada la codificación, se ha procedido a la transformación de los archivos XML en HTML, a través de una hoja de estilo específica para el proyecto, y a la migración de los contenidos en la aplicación web.

\subsection{Visualización de la web y funcionalidades}

En la parte izquierda de la pantalla de inicio de página web del proyecto de La dama boba: edición crítica y archivo digital ${ }^{20}$, aparece un índice de navegación que permite el acceso a distintas pestañas: desde la información general relativa al proyecto, hasta la bibliografía

\footnotetext{
${ }^{20}$ La página web del proyecto La dama boba: edición crítica y archivo digital puede consultarse en el enlace http://damaboba.unibo.it/.
} 
utilizada. En el apartado Estudio, se ofrecen datos relevantes sobre el argumento, la versificación y segmentación de la comedia, un detallado análisis de la transmisión textual de la misma y los criterios que se han seguido para el establecimiento del texto crítico. Asimismo, es posible descargar una versión en formato PDF tanto del texto crítico, junto con el aparato de variantes y las notas de comentario, como del apéndice lingüístico.

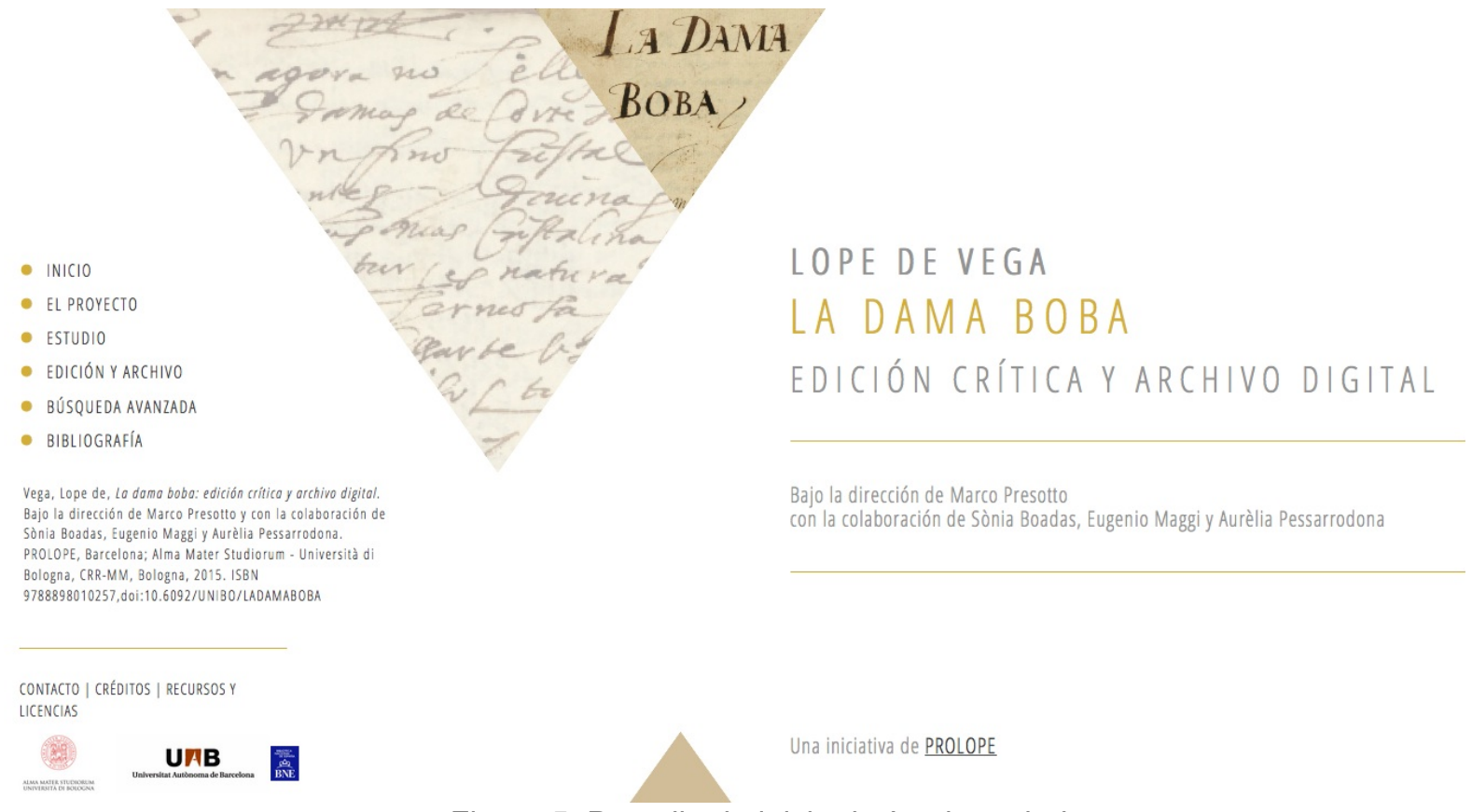

Figura 5. Pantalla de inicio de La dama boba.

Por lo que se refiere a las licencias, el proyecto se inserta dentro del marco de la Declaración de Berlín a favor del acceso abierto a los resultados de la investigación científica, según la política de la Università di Bologna, que promociona y coordina su realización. De esta manera, el producto se ha publicado bajo la licencia Creative Commons para favorecer la mayor circulación posible y la reutilización de los resultados de la investigación.

Si se accede al apartado Edición y archivo, el usuario puede consultar la aplicación web que permite visualizar cada uno de los testimonios que se han tenido en cuenta para realizar la edición crítica -autógrafo $(\mathrm{O})$, copia de memorión $(\mathrm{M})$ e impreso $(\mathrm{A})$-. Estos documentos pueden visualizarse en versión facsímil o a través de su transcripción. Además, el botón info, situado en la parte superior derecha de cada uno de los módulos, ofrece información específica sobre los testimonios. 


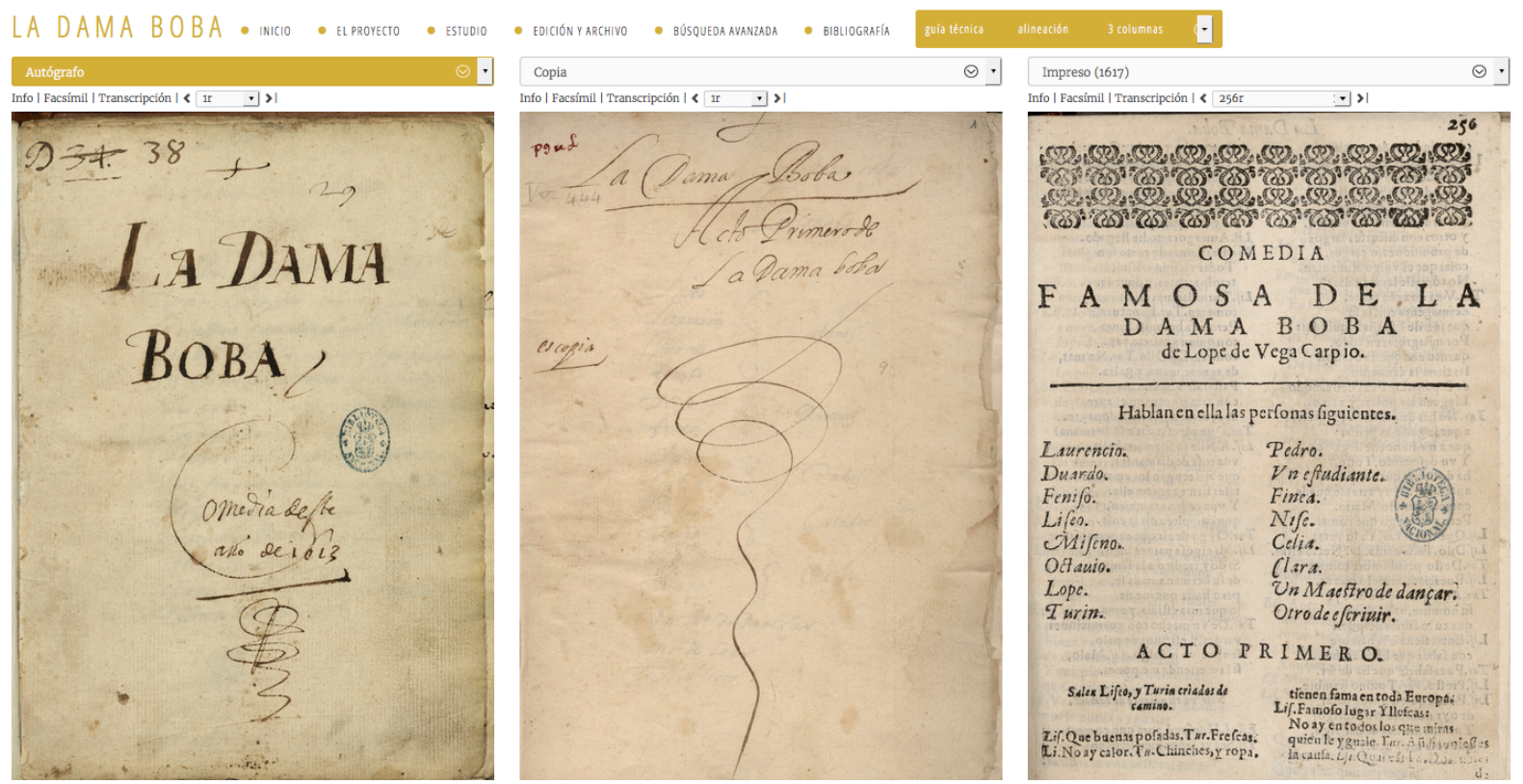

Figura 6. Visualización sinóptica de los tres testimonios en versión facsímil.

A través de la opción 3 columnas que tenemos arriba a la derecha, el usuario podrá elegir cuántos documentos quiere ver al mismo tiempo, con las opciones de tres, dos y un solo módulo. De esta manera se podrán consultar desde tres documentos sinópticamente o hasta uno solo de manera singular, ya sea en versión facsímil o consultando la transcripción de los textos.
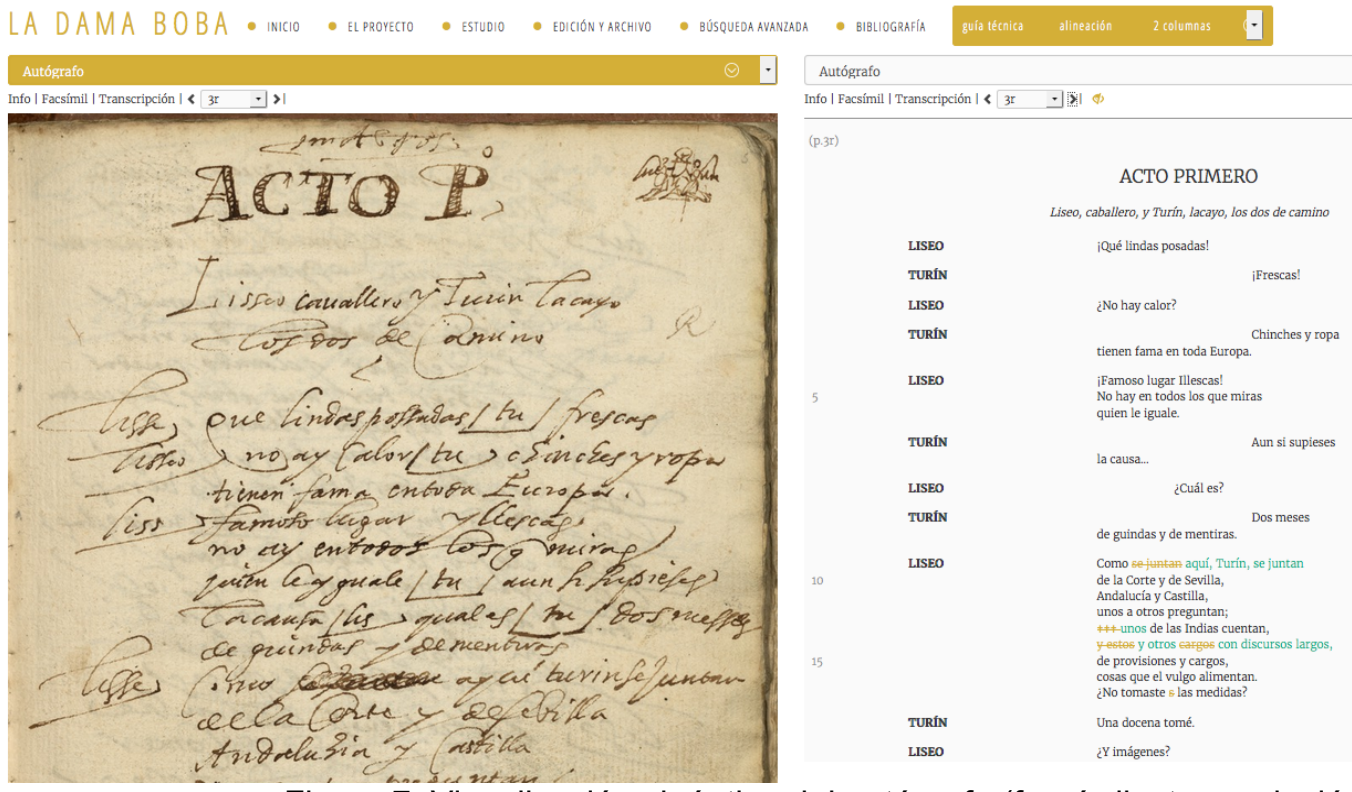

Figura 7. Visualización sinóptica del autógrafo (facsímil y transcripción).

Cuando la visualización sea de más de un texto, el usuario deberá seleccionar qué testimonio será el documento maestro, que ocupará el primer módulo a la izquierda y a partir del cual se podrá alinear el resto de textos con la opción alineación, que aparece en la parte 
superior derecha. Seleccionando esta función, los facsímiles de los testimonios, o las transcripciones de los mismos -según lo que se haya elegido-, se alinearán automáticamente con el folio que se esté consultando del documento maestro, de manera que se podrá localizar, visualizar y cotejar rápidamente un locus concreto en los tres testimonios, comprobando las variantes de la tradición textual. Una línea de color indicará el lugar donde se inicia el texto del documento maestro en los dos módulos restantes seleccionados.
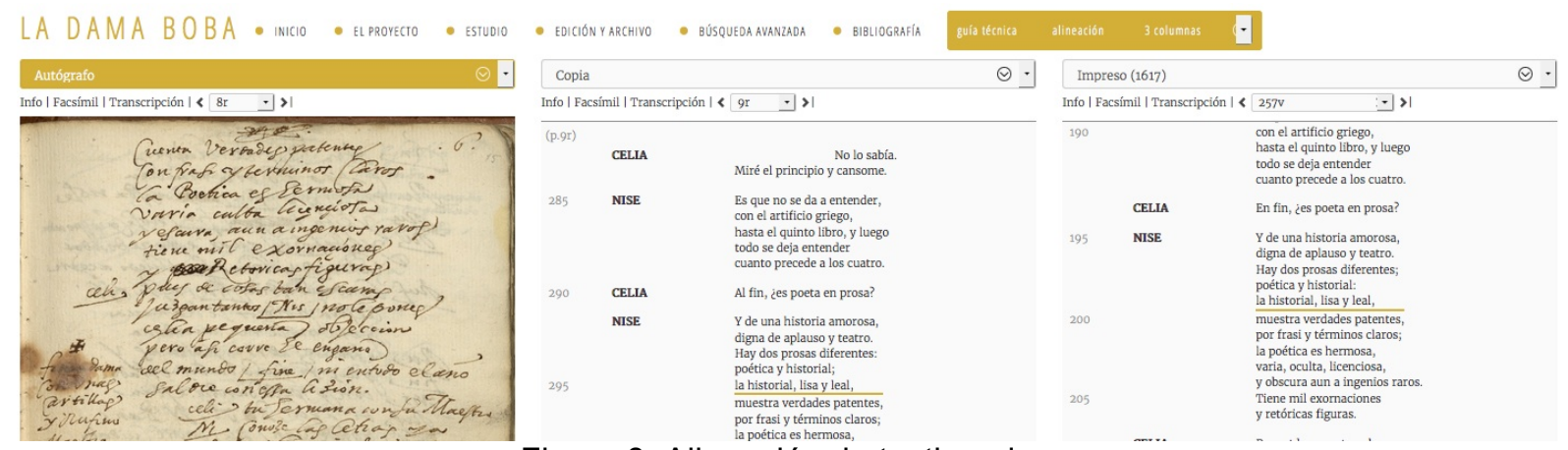

Figura 8. Alineación de testimonios.

Las variantes de la tradición textual se podrán consultar también a través de la edición crítica, a la que se puede acceder seleccionando la opción correspondiente en el desplegable de la parte superior de cada módulo.

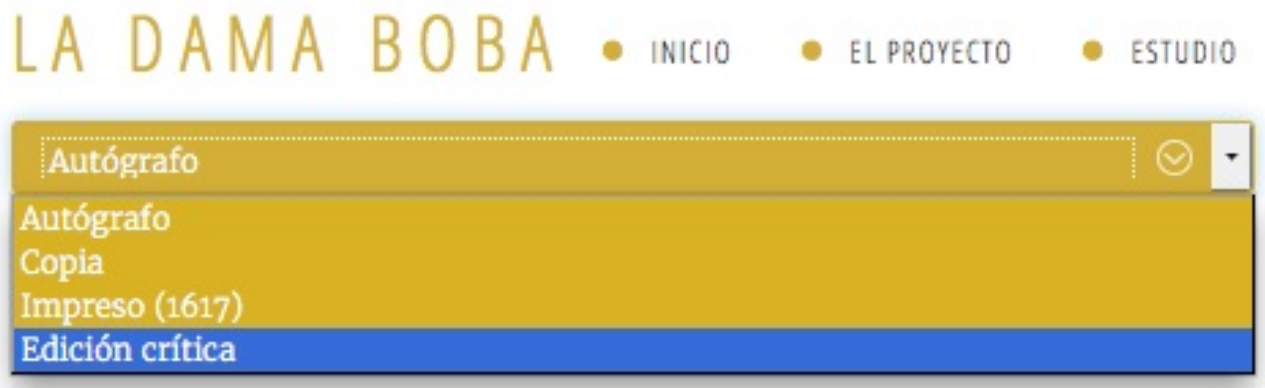

Figura 9. Opción edición crítica.

En el texto crítico los fragmentos con variantes aparecen resaltados en color oromarrón. Al pasar el cursor por encima, la información ecdótica se presenta en un pop-up. Lo mismo ocurre en el caso de variantes más complejas y que engloban varios versos. Asimismo, los asteriscos que aparecen al final de algunas líneas remiten a las notas de comentario, que complementan el texto y ayudan a su mejor comprensión. 


LA DAMA BOBA • inicio - elproyecto - estudio
Edición crítica
Info I Facsímil I Transcripción $|<3 \mathrm{r}->>|$

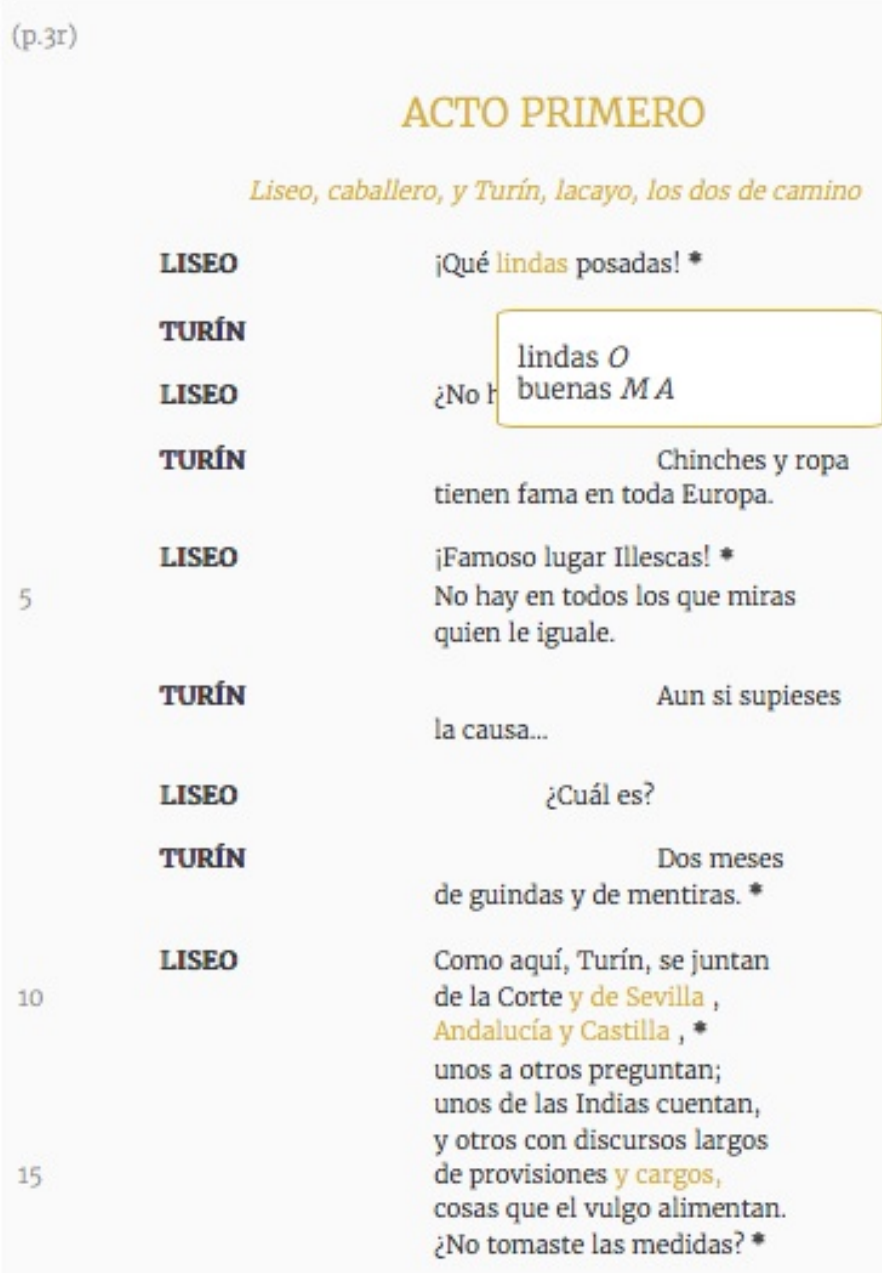

Figura 10. Visualización de variantes ecdóticas en la edición crítica.

En cuanto al autógrafo, la aplicación ofrece dos posibilidades de visualización: es posible representar todas las características del testimonio (tachaduras, sustituciones, fragmentos añadidos) para apreciar el proceso de escritura de la obra, o bien leer la transcripción del texto definitivo aprobado por el autor. En estos casos, y cuando es posible, se indica una hipótesis relativa a la secuencia con la que se produjo la intervención: en los casos más sencillos, la sucesión se evidencia con el uso de colores -oro-marrón para lo que aparece tachado en el manuscrito y verde para lo que se añade-, mientras que en los más complicados se hace uso de una animación que ejemplifica visualmente cuál ha sido el proceso de escritura. 

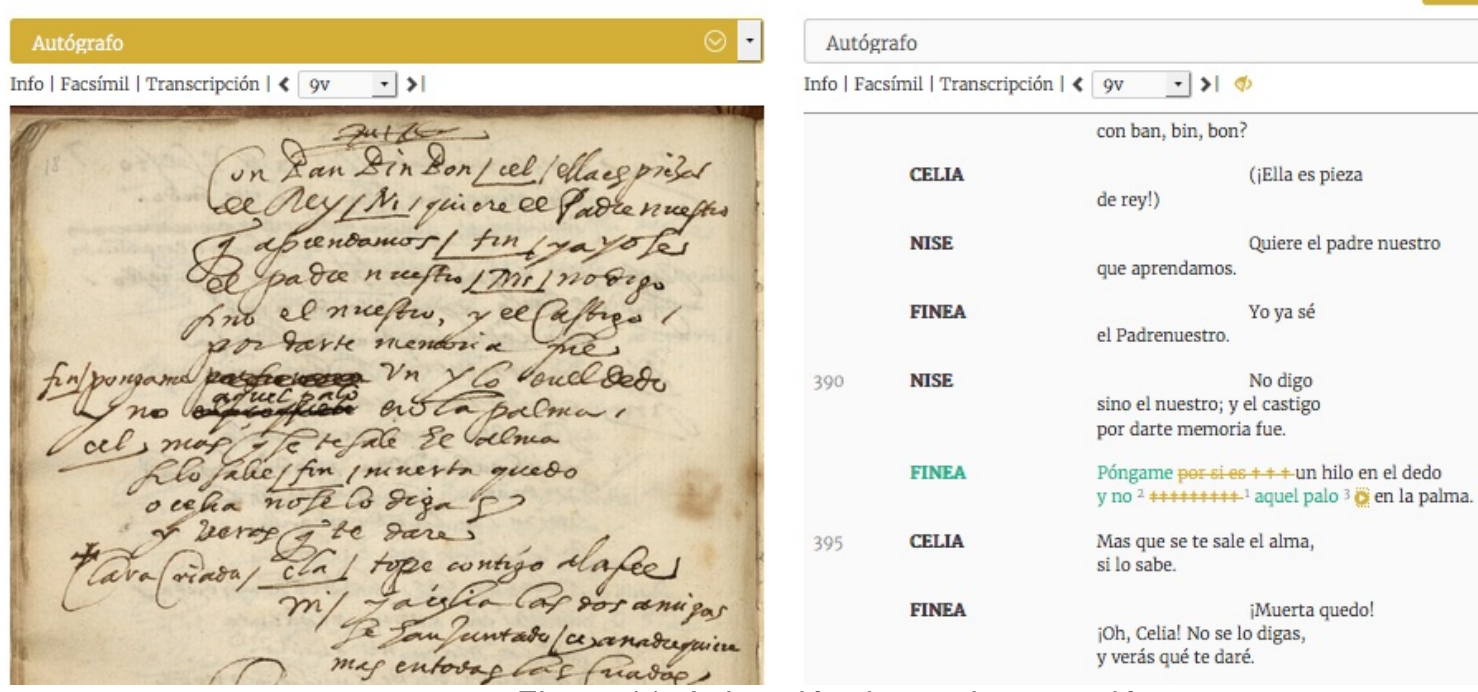

Figura 11. Animación de una intervención.

Por otra parte, el sistema de búsqueda, que encontramos en la pestaña búsqueda avanzada del menú inicio, permite acceder a todos los documentos publicados. A través de los filtros que se ofrecen, es posible seleccionar un documento específico y limitar la búsqueda al parlamento de un personaje concreto. Además, si se selecciona uno de los resultados de búsqueda, la aplicación nos remite a la página donde aparece, que podemos consultar en versión facsímil o bien en su transcripción.

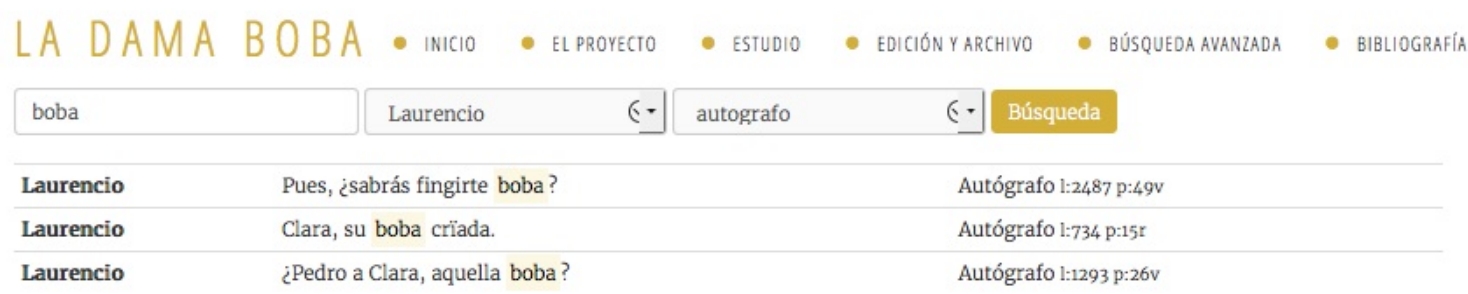

Figura 12. Opción búsqueda avanzada.

El usuario que acceda por primera vez a la página web, encontrará una explicación general del funcionamiento de la aplicación en la opción guía técnica que aparece en la parte superior derecha de la pantalla: mediante unos simples pasos y de manera muy gráfica se facilita la información práctica para su uso.

Esta versión de la edición crítica y archivo digital todavía precisa de la mejora de algunas de sus funcionalidades, en lo que el equipo técnico prevé trabajar para alcanzar un acceso más ágil a la aplicación. En cuanto a los contenidos, los editores están reelaborando las notas complementarias de la edición crítica para conseguir un doble nivel de anotación según el modelo de las ediciones de la Biblioteca Clásica de la Real Academia Española.

Estamos ante un proyecto experimental que pretende aplicar las posibilidades que ofrecen las Humanidades Digitales a la Filología, poniendo el centro de atención en los 
problemas relacionados con la tradición textual del Teatro del Siglo de Oro, que en su conjunto constituye uno de los patrimonios artísticos, más extensos y relevantes de nuestra cultura clásica. Los autógrafos teatrales, las copias manuscritas, los impresos realizados por el mismo autor, así como las ediciones sueltas, las no autorizadas o pirateadas, constituyen una documentación altamente significativa de esta cultura, cuya materialidad ayuda de manera insustituible a comprender aspectos de la historia del teatro representado, de la literatura dramática pero también de la historia del libro y de la lectura. Para ello, resulta muy oportuno explorar, gracias al soporte digital, los modelos posibles de representación de esta complejidad codicológica, bibliográfica y ecdótica. La imponente cantidad de trabajo que ha significado la realización de la aplicación, destinada a un usuario especializado y abierta a desarrollos futuros, se justifica con la ambición de crear un laboratorio de reflexión en torno a esta necesidad de la comunidad científica, a partir de algunas soluciones técnicas concretas. También el proyecto ha constituido un momento importante de formación para afinar los instrumentos de realización de las ediciones críticas digitales que representan, a nuestro modo de ver, una asignatura pendiente e ineludible del equipo Prolope y, en general, de los estudios de Filología Hispánica.

\section{REFERENCIAS BIBLIOGRÁFICAS}

BOADAS, S. y VALDÉS, R. (coords.) (2014). Lope de Vega: La famosa comedia de mujeres y criados. Barcelona: Universitat Autònoma de Barcelona. Recuperado de http://online.fliphtml5.com/tbai/hyew/ - p=8 el 22/09/2017.

FERRER VALLS, T. (dir.) (2015). María de Zayas: La traición en la amistad. Valencia: Grupo Te@doc, Universitat de València. Recuperado de http://dicat.uv.es/te@doc/edicion/TraicionenAmistad Zayas.html el 22/09/2017.

O’ NEILL, J. (ed.) (2007). La entretenida by Miguel de Cervantes: A Digital, Annotated Edition and an English Translation (The Diversion). Londres: King's College London. Recuperado de http://entretenida.outofthewings.org/aboutproject/index.html el 22/09/2017.

PRESOTTO, M. (dir.) (2015). Vega, Lope de. La dama boba: edición crítica y archivo digital. Barcelona-Bologna: PROLOPE, Universitat Autònoma de Barcelona, CRM-MM, Università di Bologna. Recuperado de http://damaboba.unibo.it/ el 22/09/2017.

SEVILLA ARROYO, F. (ed.) (2001). Miguel de Cervantes Saavedra. La Numancia. Alicante: Biblioteca Virtual Miguel de Cervantes. Recuperado de http://www.cervantesvirtual.com/obra/la-numancia--0/ el 22/09/2017. 
SOLER SASERA, E. (ed.) (2013-2014). Félix Lope de Vega y Carpio: La dama boba. Edición digital de A. Zamora Vicente, Lope de Vega: La dama boba. Madrid, Espasa-Calpe, 1946. En Biblioteca digital Artelope, J. Oleza Simó (dir.). Recuperado de http://artelope.uv.es/biblioteca/textosAL/AL0575 LaDamaBoba.php el 22/09/2017.

SUTHERLAND, K. (dir.) (2010). Jane Austen's Fiction Manuscripts: A Digital Edition. OxfordLondres: University of Oxford-King's College London. Recuperado de http://www.janeausten.ac.uk/about/overview.html el 22/09/2017.

ZAMORA VICENTE, A. (ed.) (1946). Lope de Vega: La dama boba. Madrid: Espasa Calpe. Recuperado de http://www.cervantesvirtual.com/obra/la-dama-boba--0/ el 22/09/2017. 